\title{
Burkholderia pseudomallei aerosol infection results in differential inflammatory responses in BALB/c and $\mathrm{C} 57 \mathrm{Bl} / 6$ mice
}

Correspondence

Gek-Yen Gladys Tan

tgekyen@dso.org.sg

Received 29 August 2007

Accepted 13 December 2007

\author{
Gek-Yen Gladys Tan, ${ }^{1}$ Yichun Liu, ${ }^{1}$ Suppiah Paramalingal Sivalingam, ${ }^{1}$ \\ Siew-Hoon Sim, ${ }^{1}$ Dongling Wang, ${ }^{1}$ Jean-Charles Paucod, ${ }^{2}$ Yves Gauthier $^{2}$ \\ and Eng-Eong $\mathrm{Ooi}^{1}$ \\ ${ }^{1}$ Medical Countermeasures Laboratory, DSO National Laboratories, Singapore 117510 \\ ${ }^{2}$ Centre de Recherches du Service de Santé des Armées Emile Pardé, La Tronche, France
}

\begin{abstract}
Melioidosis is caused by the Gram-negative bacterium Burkholderia pseudomallei, whose portals of entry into the body include subcutaneous, ingestion and inhalation routes. Animal models play an important role in furthering our understanding of this disease, which is associated with high morbidity and mortality in susceptible subjects. Previous studies using intranasal inoculation showed a differential susceptibility to inhalational melioidosis in BALB/c and C57BI/6 mice and attributed the difference to genetic factors and host response. However, a recent study found no difference in susceptibility when the two species of mice were exposed to nebulized bacteria. We sought to address this discrepancy by using a nasal route only, instead of whole-body aerosol exposure system. Employing three different clinical strains of B. pseudomallei and following the progression of disease development in both BALB/c and C57BI/6 mice, we found that $\mathrm{BALB} / \mathrm{c}$ mice were at least 10- to 100-fold more susceptible to infection than C57BI/6 mice. Comparison of bacterial burdens in aerosol-challenged mice, at both the pulmonary and distant sites of infection, suggests that $\mathrm{C} 57 \mathrm{BI} / 6$ mice were more efficient in clearing the bacteria than BALB/c mice. In addition, a comprehensive study of a wide panel of chemokines and cytokines at the protein level demonstrated that hyperproduction of proinflammatory cytokines in aerosol-challenged BALB/c mice did not translate into better protection and survival of these mice, whereas a moderate increase in these proteins in aerosol-challenged C57BI/6 mice was more beneficial in clearing the infection. This suggests that high levels of proinflammatory cytokines are detrimental and contribute to the immunopathogenesis of the infection.
\end{abstract}

\section{INTRODUCTION}

Burkholderia pseudomallei is the causative agent of melioidosis, a disease that is endemic in South-East Asia and northern Australia. Human infection results in many possible clinical manifestations (Wiersinga et al., 2006), with pneumonia being the most common presentation in approximately half of all reported cases (Cheng \& Currie, 2005). Though lung involvement is likely to be due to haematogenous spread following percutaneous inoculation, it is likely that, under certain environmental conditions, inhalation is a significant mode of infection (Currie, 2003). This is evidenced by reports of soldiers exposed to dust from

Abbreviations: AID, actual inhaled dose; GM-CSF, granulocyte-macrophage colony-stimulating factor; IFN, interferon; IL, interleukin; KC, keratinocyte-derived chemokine; MCP, monocyte-chemotactic protein; MIG, monokine induced by gamma interferon; MIP, macrophage inflammatory protein; TID, theoretical inhaled dose; TNF, tumour necrosis factor. helicopter rotor downwash in Vietnam (Howe et al., 1971) and the association between heavy rainfalls and increased incidence of pneumonic and septicaemic presentations with poor clinical outcomes (Cheng et al., 2006; Currie, 2003; Currie \& Jacups, 2003; Liu et al., 2006). Furthermore, B. pseudomalle $i$ is also a potential bioterror agent and the most likely mode of dissemination during a biological attack is through an infectious aerosol. It is thus important to understand the pathogenesis of melioidosis following an inhalational exposure to B. pseudomallei.

Currently, by adopting artificial routes of infection, many research groups have demonstrated that differential susceptibility to $B$. pseudomallei infection exists between $\mathrm{BALB} / \mathrm{c}$ and $\mathrm{C} 57 \mathrm{Bl} / 6$ mice, with the latter being more resistant (Hoppe et al., 1999; Leakey et al., 1998; Liu et al., 2002). However, Jeddeloh et al. (2003) reported that the two strains of mice displayed similar sensitivities to nebulized B. pseudomallei and ruled out the contribution made by genetic differences in the host to the disease. This 
finding suggests that an exposure to aerosolized B. pseudomallei results in fundamentally different pathogenesis and disease outcome, compared with inoculation by intranasal droplets of bacterial culture. Should this observation be correct, it would invalidate many previous studies on the pathogenesis of inhalational melioidosis. Jeddeloh et al. (2003), however, had used a whole-body aerosol system, which could have resulted in deposition of bacteria on the animals in addition to entry into the lungs. In order to provide an accurate model for pneumonic melioidosis in humans and to resolve the current discrepancy in the various murine models, we established a nose-only aerosol infection system to characterize accurately the early events that occur in post-inhalational challenge. Furthermore, we also characterized for the first time the induction of 17 chemokine and cytokine responses at the protein level.

\section{METHODS}

Animals. Female 4- to 8-week-old $\mathrm{BALB} / \mathrm{c}$ and $\mathrm{C} 57 \mathrm{Bl} / 6$ mice were purchased from the Center for Animal Resources of the National University of Singapore and housed in polystyrene cages with a bedding of wood shavings. The mice were fed with commercial pellets (PMI Nutrition International) and potable water ad libitum. The cages were placed in a micro-isolator within the BioSafety Level Three facility. All experimental procedures with mice and infection were approved by the Animal Care and Use Committee, DSO National Laboratories.

Bacteria. Three different strains of B. pseudomallei (KHW, EB6103 and K96243) were used in this study. KHW was isolated from a 19-year-old Singapore national serviceman who died from melioidosis in 1989. EB6103 was isolated from a 19-year-old Singaporean male who succumbed to melioidosis in the 2004 outbreak (Liu et al., 2006). K96243 was isolated from a 34-year-old Thai diabetic woman in 1996. For infection experiments, bacteria were cultured on trypticase soy agar (TSA; BD Diagnostics) for $24 \mathrm{~h}$ at $37{ }^{\circ} \mathrm{C}$. Colonies were resuspended in sterile $\mathrm{PBS}$ to an $\mathrm{OD}_{600}$ of 1.9 (equivalent to $1 \times 10^{9}$ c.f.u. $\mathrm{ml}^{-1}$ ). The bacterial suspension was diluted to the desired concentrations for inoculation into mice and aliquots of these suspensions were plated on TSA in duplicate for retrospective determination of the number of viable bacteria inoculated into the mice.

Aerosol generation. The aerosol chamber was housed within a flexiglass glove box. Bacterial suspensions of $10^{5}-10^{9}$ c.f.u. $\mathrm{ml}^{-1}$ in PBS were placed in three collision air-jet nebulizers. Nebulization of each suspension was driven by compressed air at a pressure of 1-1.5 bar for $10 \mathrm{~min}$ to generate aerosol particles of size $1-5 \mu \mathrm{m}$. Aerosol density was measured by bubbling $10 \mathrm{l}$ of the air in the aerosol chamber into $20 \mathrm{ml}$ PBS in the impinger. Aliquots of the suspensions were diluted and plated in duplicate on TSA for viable counts. Good linear correlation between the bacterial concentrations in PBS (c.f.u. $\mathrm{ml}^{-1}$ ) and bacterial density (c.f.u. per litre of air) in the aerosols was observed and the results were highly reproducible. Enumeration of viable B. pseudomallei showed that the bacterial counts in the aerosol were consistently $10^{3}$-fold lower than those in the corresponding bacterial suspensions.

Aerosol infection of mice. Mice were restrained in $50 \mathrm{ml}$ Falcon tubes in the aerosol chamber and exposed in a nose-only manner to the aerosols of $B$. pseudomallei for $30 \mathrm{~min}$. To determine the actual inhaled dose (AID) after aerosol challenge, three mice from each group were sacrificed immediately post-exposure, and their lungs were aseptically removed. The actual number of bacteria that entered the lungs was determined as described below. The theoretical inhaled dose (TID) was calculated according to the method of Guyton (1947). Control mice were subjected to aerosols of PBS.

Intranasal, intraperitoneal and subcutaneous infection of mice. For intranasal inoculation of bacteria, the mice were lightly anaesthetized with $3 \%$ isoflurane in oxygen. The desired dose of bacteria was then delivered through one nostril of each mouse with a pipette tip in a total volume of $20 \mu \mathrm{l}$ PBS. For intraperitoneal infection of mice, the appropriate dose of bacteria in a total volume of $100 \mu \mathrm{l}$ PBS was injected while, for subcutaneous infection, a volume of $50 \mu \mathrm{l}$ PBS containing the appropriate number of bacteria was injected at the left inguinal location of the mice. Control mice received PBS only.

Determination of $\mathbf{L D}_{\mathbf{5 0}}$. Ten- and 20 -day $\mathrm{LD}_{50}$ values were determined by the method of Reed \& Muench (1938). Mice were divided into six groups of six per group. Five different doses of bacteria were used to infect the mice and the last group received PBS as a control. For aerosol experiments, the AID was used for the calculation of $\mathrm{LD}_{50}$ values whereas, for intranasal, intraperitoneal and subcutaneous experiments, the concentration of bacterial suspensions used for infection of the mice were adopted for the $\mathrm{LD}_{50}$ value calculation.

Enumeration of viable B. pseudomallei in organs, nasal cavity and blood. After aerosol infection with a low dose of B. pseudomallei, three mice were euthanized 1, 3 and 6 days post-challenge. Blood was collected aseptically in lithium heparin by cardiac puncture. The nasal cavity was washed with $1 \mathrm{ml}$ sterile PBS. Lungs, spleens and livers were removed aseptically and placed in 2 or $4 \mathrm{ml}$ sterile PBS. The organs were homogenized through a $100 \mu \mathrm{m}$ nylon mesh sieve in a 6well plate, after which they were serially diluted and plated in duplicate on Ashdown's agar plates (Ashdown, 1979). After incubation at $37{ }^{\circ} \mathrm{C}$ for $48 \mathrm{~h}$, colonies were counted from plates of appropriate dilutions and the mean colony count was derived to give the bacterial load in the different organs.

Measurement of cytokine and chemokine production. Lung and spleen homogenates from aerosol-infected mice were spun at $18000 \mathrm{~g}$ for $5 \mathrm{~min}$ in a Microfuge 18 centrifuge (Beckman Coulter) before they were filtered through a $0.22 \mu \mathrm{m}$ Millex-GV filter unit (Millipore). The homogenates were then assayed for gamma interferon (IFN- $\gamma$ ), tumour necrosis factor (TNF)- $\alpha$, interleukin (IL)-2, IL-3, IL-4, IL-5, IL-6, IL-9, IL-10, IL-12p70, IL-13, granulocyte-macrophage colony-stimulating factor (GM-CSF), monocyte-chemotactic protein (MCP)-1, keratinocyte-derived chemokine $(\mathrm{KC})$, monokine induced by gamma interferon (MIG), macrophage inflammatory protein (MIP)-1 $\beta$ and RANTES (CCL5) using the FACS Array Bioanalyser (BD Biosciences) and CBA Flex Set System, according to the manufacturer's instructions. Samples from lungs and spleens of uninfected mice were tested as negative controls to obtain cut-off values. The mean values $\left(\mathrm{pg} \mathrm{ml}^{-1}\right)$ from three negative control mice (BALB/c/C57Bl/6) are as follows: (lungs) TNF- $\alpha$, $0 / 21$; IFN- $\gamma$, 0/1; IL-6, 0/3; KC, 2/2; RANTES, 249/263; MCP-1, 0/27; MIG, 35/19; MIP-1 $\beta$, 8/9; IL-10, 39/36; (spleens) TNF- $\alpha, 41 / 0$; IFN- $\gamma, 4 /$ 0; IL-6, 6/0; KC, 4/0; RANTES, 4284/3728; MCP-1, 55/0; MIG, 94/91; MIP-1 $\beta, 29 / 23$; IL-10, 60/41. The assays were done in duplicate.

Statistical analysis. Data were analysed using Student's unpaired $t$ test; $P<0.05$ was considered to be significant.

\section{RESULTS AND DISCUSSION}

\section{Differential susceptibility of BALB/C and C57BI/6 mice to $B$. pseudomallei via different routes of infection}

To mimic the different ways in which humans may acquire melioidosis, both $\mathrm{BALB} / \mathrm{c}$ and $\mathrm{C} 57 \mathrm{Bl} / 6$ mice were infected 
with three different strains of $B$. pseudomallei via intranasal (infection through inhalation in humans), intraperitoneal (systemic melioidosis) or subcutaneous (percutaneous inoculation) routes. Mice were monitored daily to follow their progression towards severe disease/mortality. BALB/c mice infected with any of the three strains via the intranasal route succumbed to the disease within $48 \mathrm{~h}$ of challenge, and represented an acute form of melioidosis, whereas those infected via the intraperitoneal route only displayed disease symptoms from day 6 post-challenge. For BALB/c mice infected subcutaneously, the onset of disease only appeared on day 23 post-infection. A similar trend in the development of disease via the intranasal, intraperitoneal and subcutaneous routes of infection was observed with the $\mathrm{C} 57 \mathrm{Bl} / 6$ mice, with the main difference being a much delayed onset of disease. Hence, acute and chronic forms of melioidosis are established in mice infected intranasally and subcutaneously, respectively.

Table 1 summarizes the $\mathrm{LD}_{50}$ values obtained for the different routes of infection in both strains of mice with the three strains of bacterium. The data unambiguously demonstrated that BALB/c mice were more susceptible to infection by all strains via the different portals of entry, than the $\mathrm{C} 57 \mathrm{Bl} / 6$ mice, by a factor of 10 - to 100 -fold. Our results are in accordance with studies performed by various groups for the intranasal, intravenous, intraperitoneal and subcutaneous routes (Barnes \& Ketheesan, 2005; Hoppe et al., 1999; Liu et al., 2002). Collectively, from day 10 (intranasal and intraperitoneal) and day 20 (intraperitoneal and subcutaneous) $\mathrm{LD}_{50}$ data, the order of the lethality of the route of infection, in decreasing order, was intranasal $>$ intraperitoneal $>$ subcutaneous. Furthermore, B. pseudomallei strain KHW was the most virulent of the three strains tested. Hence, variations in the genetic background of hosts and bacterial strains encountered in the environment, as well as the different portals of entry, are important factors and contribute to the varying disease outcomes.

\section{Infection via aerosolization mimics closely the inhalational route in humans}

Infection of mice via aerosolization was examined in order to establish an accurate murine model that more closely mimics the inhalational route in humans. B. pseudomallei strain KHW was chosen for this route owing to its increased virulence demonstrated above. Mice were infected with a nebulized suspension in a nose-only exposure system. The $\mathrm{LD}_{50}$ values obtained for $\mathrm{BALB} / \mathrm{c}$ and $\mathrm{C} 57 \mathrm{Bl} / 6$ mice were $<20$ and 423 c.f.u., respectively (Table 1 ); $\mathrm{C} 57 \mathrm{Bl} / 6$ mice were therefore at least 20 -fold more resistant to nebulized $B$. pseudomallei than $\mathrm{BALB} / \mathrm{c}$ mice. This contrasts with the results obtained by Jeddeloh et al. (2003) using a whole-body aerosol exposure system in which both BALB/c $\left(\mathrm{LD}_{50} 10\right.$ c.f.u.) and $\mathrm{C} 57 \mathrm{Bl} / 6\left(\mathrm{LD}_{50} 27\right.$ c.f.u.) mice exhibited similar sensitivities to nebulized B. pseudomallei strain 1026b. The discrepancy in these results is most likely due to the utilization of different aerosol exposure systems in the two studies. In a whole-body aerosol exposure system, nebulized B. pseudomallei are not only inhaled through the nose, but also through the mouth, and a large proportion of the bacteria would also be trapped in body fur. Subsequently, following aerosol exposure, ingestion or inhalation of additional nebulized bacteria on the fur could occur, leading to much higher exposure doses than would otherwise be accounted for. Hence, non-pulmonary routes of infection may complicate the infection. In contrast, in a nose-only aerosol exposure system, the mice are subjected to the nebulized bacteria only during the actual duration of aerosolization. Hence, the number of bacteria delivered to the mice can be accurately determined. Bearing in mind that the bacterial strains used in the two studies are highly virulent, the addition of even a few bacteria would be enough to induce a very different clinical outcome. Furthermore, the $\mathrm{LD}_{50}$ values obtained by Jeddeloh et al. (2003)were based on the TID values, whereas in our system, the $\mathrm{LD}_{50}$ values were based on the AID values in the mice. The TID was derived on

Table 1. Comparison of 10 day and/or 20 day $\mathrm{LD}_{50}$ values (c.f.u.) of $\mathrm{BALB} / \mathrm{c}$ and $\mathrm{C} 57 \mathrm{BI} / 6$ mice infected with three strains of $B$. pseudomallei via four different routes

Both strains of mice were infected with strains KHW, EB6103 or K96243 via aerosol, intranasal, intraperitoneal or subcutaneous routes. Mice were monitored daily for progression of disease for 10 days (aerosol and intranasal) or 20 days (intraperitoneal and subcutaneous). Mortality of the infected mice was scored and $\mathrm{LD}_{50}$ values were calculated. For aerosolization experiments, the AID was used for the calculation of $\mathrm{LD}_{50}$ values; for intranasal, intraperitoneal and subcutaneous experiments, the concentration of bacterial suspensions used for infection of the mice were adopted for the $\mathrm{LD}_{50}$ value calculation. Aerosolization and intranasal experiments were performed twice and similar values were obtained. ND, Not done.

\begin{tabular}{|c|c|c|c|c|c|c|}
\hline \multirow[t]{2}{*}{ Route of infection } & \multicolumn{2}{|c|}{ KHW (c.f.u.) } & \multicolumn{2}{|c|}{ EB6103 (c.f.u.) } & \multicolumn{2}{|c|}{ K96243 (c.f.u.) } \\
\hline & $\mathrm{BALB} / \mathrm{c}$ & $\mathrm{C} 57 \mathrm{Bl} / 6$ & $\mathrm{BALB} / \mathrm{c}$ & $\mathrm{C} 57 \mathrm{Bl} / 6$ & $\mathrm{BALB} / \mathrm{c}$ & $\mathrm{C} 57 \mathrm{Bl} / 6$ \\
\hline Aerosol (10 days) & $<20$ & 423 & $\mathrm{ND}$ & $\mathrm{ND}$ & $\mathrm{ND}$ & $\mathrm{ND}$ \\
\hline Intranasal (10 days) & 16 & 6230 & $>850$ & 22000 & 226 & 15240 \\
\hline \multicolumn{7}{|l|}{ Intraperitoneal } \\
\hline 10 days & 1100 & $>58750$ & 33920 & $>8.5 \times 10^{5}$ & 54063 & $5.3 \times 10^{6}$ \\
\hline 20 days & 378 & $>58750$ & 3680 & $>8.5 \times 10^{5}$ & 43375 & $5.3 \times 10^{6}$ \\
\hline Subcutaneous (20 days) & $5 \times 10^{5}$ & $2.3 \times 10^{7}$ & $1.3 \times 10^{6}$ & $4.7 \times 10^{7}$ & $3 \times 10^{7}$ & $5.4 \times 10^{8}$ \\
\hline
\end{tabular}


Table 2. AID of viable aerosolized B. pseudomallei in the lungs of BALB/c and C57BI/6 mice

Both strains of mice were exposed to varying concentrations of nebulized B. pseudomallei strain KHW for 30 min. Immediately after aerosol exposure, three mice from each group were euthanized. Their lungs were removed aseptically and homogenized and homogenates were plated to determine the number of viable bacteria retained in the lungs. Three independent experiments were conducted for bacterial suspension of concentration $10^{5}$ c.f.u. $\mathrm{ml}^{-1}$, while experiments with bacterial suspensions of concentrations of $10^{6}-10^{9}$ c.f.u. $\mathrm{ml}^{-1}$ were performed twice independently. Results obtained from all three experiments performed under same conditions are shown here.

\begin{tabular}{|lccc|}
\hline \multirow{2}{*}{$\begin{array}{l}\text { Bacterial suspension } \\
\text { (c.f.u. } \mathbf{~ m l}^{\mathbf{- 1}} \text { ) }\end{array}$} & $\begin{array}{c}\text { Aerosol density } \\
\left.\text { (c.f.u. } \mathbf{~}^{\mathbf{- 1}}\right)\end{array}$ & \multicolumn{2}{c|}{ Mean AID (c.f.u. per pair of lungs) } \\
\cline { 3 - 4 } & & BALB/c (individual results) & C57B1/6 (individual results) \\
\hline $10^{5}$ & $10^{2}$ & $18(20,20,14)$ & $1.3(0,0,4)$ \\
$10^{6}$ & $10^{3}$ & $137(133,140)$ & $89(67,110)$ \\
$10^{7}$ & $10^{4}$ & $2212(1753,2670)$ & $847(633,1060)$ \\
$10^{8}$ & $10^{5}$ & $14289(12577,16000)$ & $8617(8553,8680)$ \\
$10^{9}$ & $10^{6}$ & $185000(196000,174000)$ & $96092(103433,88750)$ \\
\hline
\end{tabular}

the basis of the weight-dependent respiratory minute volume of the mice (Guyton, 1947), but the AID represented the actual number of nebulized bacteria inhaled by the mice and would thus be more accurate for the calculation of $\mathrm{LD}_{50}$ values. Hence, the murine model of acute pneumonic melioidosis, established by the nose-only aerosol exposure system, more closely resembles that of human pneumonic melioidosis, rather than a whole-body exposure system (Jeddeloh et al., 2003) or the intranasal route of infection (Liu et al., 2002).

Comparing the $\mathrm{LD}_{50}$ values obtained from the aerosol and intranasal routes of infection, there was no difference for $\mathrm{BALB} / \mathrm{c}$ mice infected via either inhalational route. However, for $\mathrm{C} 57 \mathrm{Bl} / 6$ mice, the day $10 \mathrm{LD}_{50}$ values were 423 and 6230 c.f.u. for the aerosol and intranasal routes of infection, respectively. These results suggest that the aerosol route of infection is more lethal than the intranasal route, and this could be explained by the fact that aerosols generated in the range of $1-5 \mu \mathrm{m}$ can enter and be retained in the lungs, whereas droplets of bacteria delivered intranasally tend to get lodged in the nasal passage. Hence, the order of lethality for the different routes of infection, in decreasing order, was aerosol $>$ intranasal $>$ intraperitoneal $>$ subcutaneous.

\section{Bacterial colonization in B. pseudomallei aerosols-infected BALB/C and C57BI/6 mice}

To investigate other factors which could contribute to the differential susceptibility observed between the two murine hosts, the kinetics of bacterial clearance or colonization in both $\mathrm{BALB} / \mathrm{c}$ and $\mathrm{C} 57 \mathrm{Bl} / 6$ mice was determined at four different time points after aerosol challenge (immediately, day 1 , day 3 and day 6). Both strains of mice were subjected to nebulized B. pseudomallei KHW with an aerosol density of 30 c.f.u. $1^{-1}$ for $30 \mathrm{~min}$. This dose was chosen because it induced $100 \%$ mortality in BALB/c mice within 10 days, but had no apparent effect on $\mathrm{C} 57 \mathrm{Bl} / 6$ mice. Colony counting at the earliest time point revealed a mean of 14 and 4 c.f.u. of B. pseudomallei detected in the lungs of $\mathrm{BALB} / \mathrm{c}$ and $\mathrm{C} 57 \mathrm{Bl} / 6$ mice, respectively. To exclude that this difference, observed at such an early time point, was not due to the low concentration of the bacterial suspension used, two separate experiments were performed using five different bacterial suspensions of $10^{5}$ to $10^{9}$ c.f.u. $\mathrm{ml}^{-1}$. Both strains of mice were subjected to each aerosol density for $30 \mathrm{~min}$. The results showed that a mean of 1.5 - to 2 -fold more viable bacteria were retained in the lungs of $\mathrm{BALB} / \mathrm{c}$ mice than those of C57BL/6 mice (Table 2). The difference in bacterial numbers observed at the earliest time point was also consistent with the kinetics of bacterial growth over days 1 , 3 and 6 following aerosol infection, in the lungs and nasal washes (i.e. the primary sites of infection), as well as the spleen, liver and blood (examined to determine the extent of bacterial dissemination) (Fig. 1). Collectively, BALB/c mice had overwhelming bacterial loads, which increased tremendously over the 6 day post-infection period, displaying marked organotropism for the lungs, spleens and livers. In contrast, $\mathrm{C} 57 \mathrm{Bl} / 6$ mice had much reduced bacterial loads, with some mice clearing the infection.

\section{Induction of cytokines and chemokines}

To characterize the early immune events which take place following aerosol challenge, both locally at the pulmonary site and systemically at distant sites, 17 cytokines and chemokines (IFN- $\gamma$, TNF- $\alpha$, IL-2, IL-3, IL-4, IL-5, IL-6, IL9, IL-10, IL-12p70, IL-13, GM-CSF, MCP-1, KC, MIG, MIP- $1 \beta$ and RANTES) were measured at the protein level in the lungs and spleens of $\mathrm{BALB} / \mathrm{c}$ and $\mathrm{C} 57 \mathrm{Bl} / 6$ mice following infection with aerosols of $B$. pseudomallei strain KHW (Fig. 2). Low levels of TNF- $\alpha$, IL-6, IFN- $\gamma, \mathrm{KC}$, RANTES, MCP-1, MIG and MIP- $1 \beta$ were detectable at day 1 post-infection in the lungs of $\mathrm{C} 57 \mathrm{Bl} / 6$ mice and they increased to a modest level at either day 3 or day 6 (Fig. 2, left column). In contrast, very high levels of most of these cytokines and chemokines were found in the lungs of BALB/c mice on days 3 and day 6 (Fig. 2, left column). A modest level of IL-10 was detected in the lungs of $\mathrm{C} 57 \mathrm{Bl} / 6$ 
$\mathrm{BALB} / \mathrm{C}$
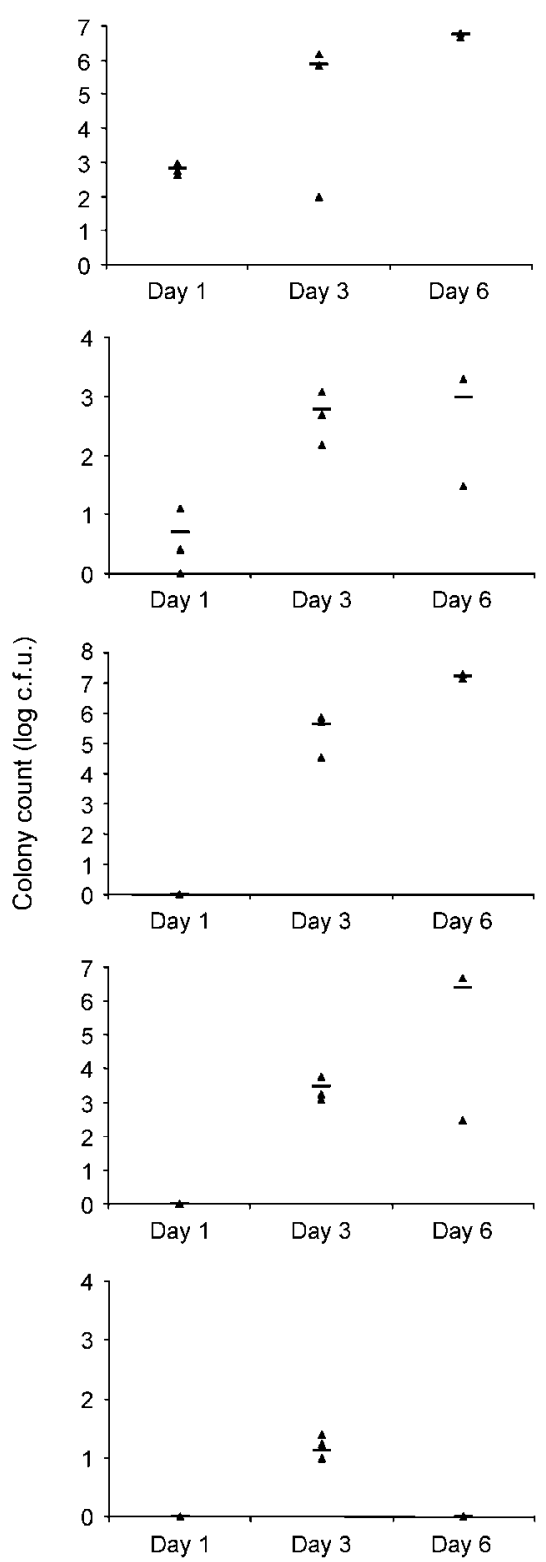

$\mathrm{C} 57 \mathrm{~B} \mid / 6$
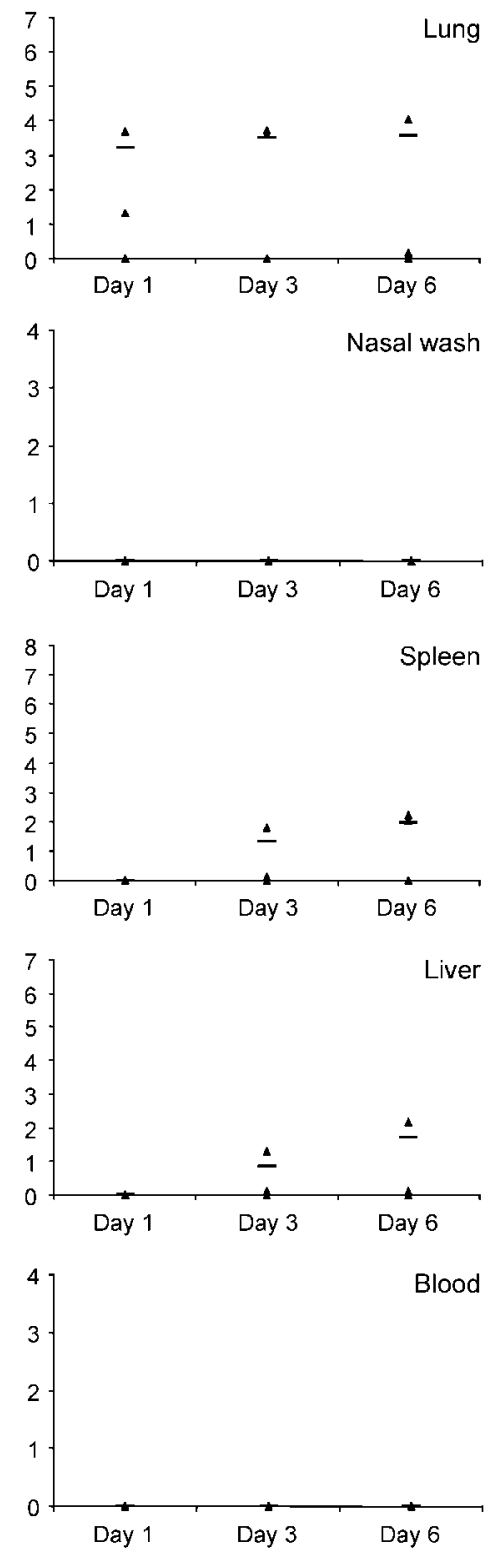

Time after exposure

Fig. 1. Bacterial colonization or clearance in organs of aerosol-infected BALB/c and C57BI/ 6 mice. Three mice per group were exposed to nebulized B. pseudomallei strain $\mathrm{KHW}$ at a density of 30 c.f.u. $\mathrm{I}^{-1}$ for $30 \mathrm{~min}$. Colony counting immediately after infection revealed means of 14 and 4 c.f.u. of B. pseudomallei in the lungs, as well as means of 12 and 1 c.f.u. of the bacterium in the nasal washes, of BALB/C and $\mathrm{C} 57 \mathrm{BI} / 6$ mice, respectively. Different cohorts of three mice per group per day were sacrificed at days 1,3 and 6 following aerosol exposure. Counts of $B$. pseudomallei c.f.u. from each mouse are represented as filled triangles, while the mean value is indicated by a bar. Values are expressed as log c.f.u. per organ. Two independent experiments were performed and similar values were obtained. Results from one experiment are shown. mice on days 1 and 6 , but it remained undetectable in the lungs of BALB/c mice on days 1, 3 and 6. Protein levels in spleens followed trends similar to those observed in the lungs of both strains of mice. GM-CSF and IL-12p70 were found to be at very low levels in both the lungs and spleens of BALB/c and C57Bl/6 mice, whereas IL-2, IL-3, IL-4, IL5 , IL9 and IL-13 were undetectable in both organs of both strains of mice (data not shown). Cellular sources of these cytokines have been reported: natural killer (NK) cells have been shown to be the principal source of IFN- $\gamma$, with contributions by $\mathrm{CD} 8^{+} \mathrm{T}$ cells through a bystander effect (Lertmemongkolchai et al., 2001); monocytes and neutrophils have been shown to be the major producers of TNF- $\alpha$ (Easton et al., 2007).
The increased protein levels of cytokines and chemokines in $\mathrm{BALB} / \mathrm{c}$ mice paralleled the corresponding increase in their bacterial loads. No cytokine polarization was found in the lungs and spleens of either $\mathrm{BALB} / \mathrm{c}$ or $\mathrm{C} 57 \mathrm{Bl} / 6$ mice, as we were able to detect levels of proinflammatory cytokines as well as IFN- $\gamma$ and chemokines. Therefore, the susceptibility of BALB/c mice to $B$. pseudomallei infection was not due to an absent or inadequate inflammatory response. Similarly, this observation has been reported by other groups using either the intranasal, intraperitoneal or intravenous models (Barnes et al., 2001; Liu et al., 2002; Ulett et al., 2000). However, data from these groups consisted mainly of mRNA responses, and not actual protein expression levels (Barnes et al., 2001; Ulett et al., 


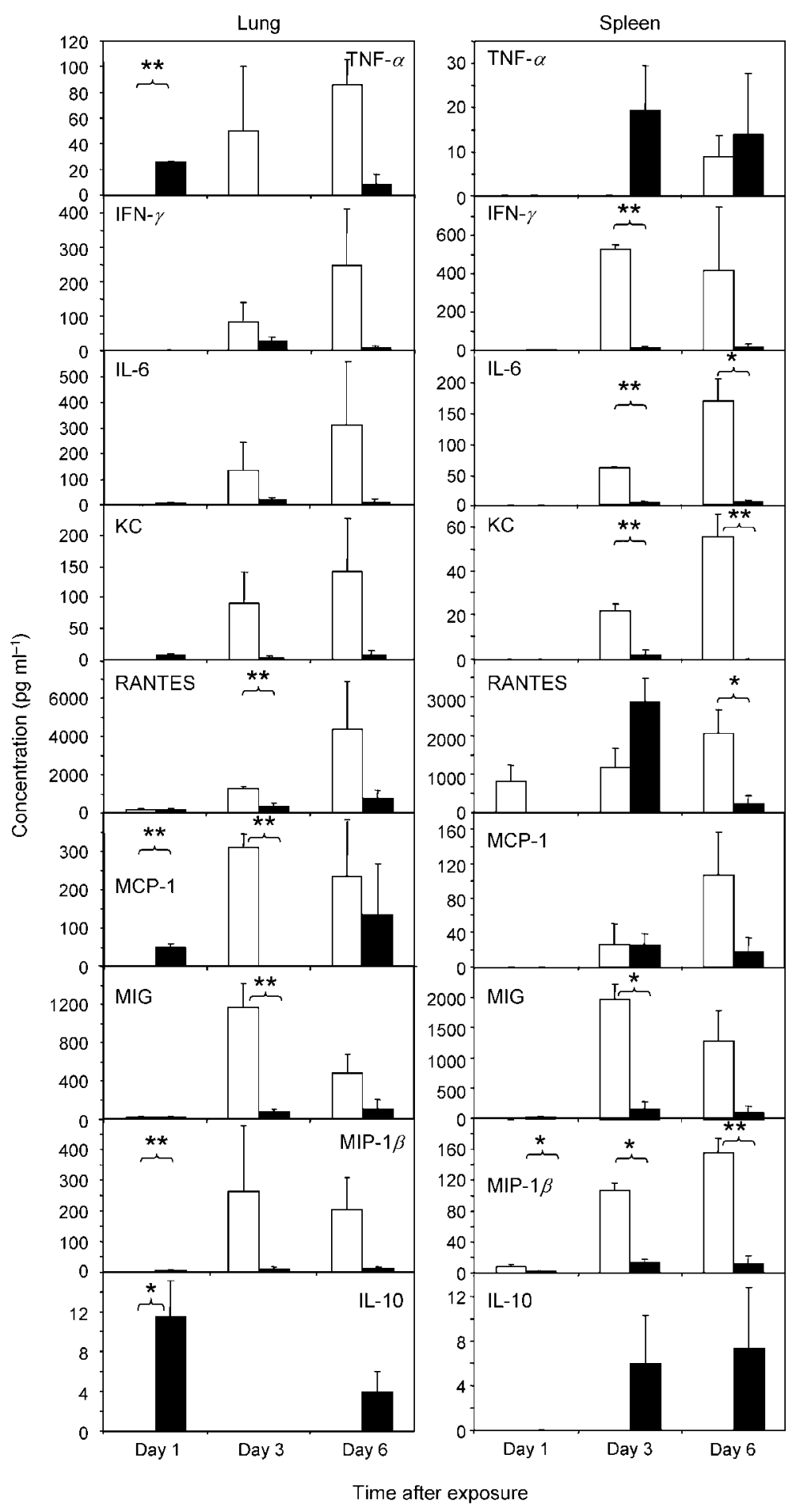

Fig. 2. Induction of cytokines and chemokines in lungs and spleens of aerosol-infected BALB/C and $\mathrm{C} 57 \mathrm{BI} / 6$ mice. Mice were infected with $\mathrm{KHW}$ aerosols of 30 c.f.u. $\mathrm{I}^{-1}$ for $30 \mathrm{~min}$. At days 1,3 and 6 post-infection, lungs and spleens from three mice per group were excised and homogenized. The lung (left column) and spleen (right column) homogenates of BALB/c (open bars) and $\mathrm{C} 57 \mathrm{BI} / 6$ (filled bars) mice were assayed for the presence of cytokines and chemokines (note the different scales in the two columns). Data shown are mean values with error bars showing the SEM from three mice at each time point. Samples from uninfected mice were tested as negative controls to obtain cut-off values. The assays were done in duplicate. Two independent experiments were performed and similar values were obtained. Results from one experiment are shown here. ${ }^{*}, P<0.05 ;{ }^{* \star}, P<0.01$.

2000). Since an increase in the transcriptional activity of cytokine and chemokine genes does not necessarily translate into release of functional protein products, our study is the first report of a comprehensive investigation of the cytokines and chemokines induced at the protein level for the nose-only aerosol infection model, which, in itself, is a better model of acute pneumonic melioidosis in humans than the intranasal model.
Both local and systemic production of IFN $-\gamma$ were higher in $\mathrm{BALB} / \mathrm{c}$ mice than in $\mathrm{C} 57 \mathrm{Bl} / 6$ mice, suggesting that hyperproduction of IFN- $\gamma$ is not protective and may lead to septic shock (Car et al., 1994; Heremans et al., 1990). Studies in patients with melioidosis have also demonstrated a link between severity of disease and the levels of cytokines and chemokines in serum, including IFN- $\gamma$, TNF- $\alpha$, IL- 6 and IL- 8 (Brown et al., 1991; Friedland et al., 1992; Lauw et al., 1999). 
Hence, the early production of local proinflammatory cytokines observed in BALB/c mice appears to spill over into the systemic compartment, and thus contribute to the immunopathogenesis of the disease (Liu et al., 2002). In contrast, $\mathrm{C} 57 \mathrm{Bl} / 6$ mice responded to the infection with a moderate and transient induction of proinflammatory response, and they were able to control and clear the infection. Although too much of a good thing, i.e. IFN- $\gamma$ in this case, is not ideal, the obligatory role of this cytokine for the survival of infected mice is undisputed (Santanirand et al., 1999). Our observation that IL-10 was only found in C57Bl/6 mice, and not $\mathrm{BALB} / \mathrm{c}$ mice, is in contrast to those reported by Koo \& Gan (2006) who observed higher IL-10 production by $\mathrm{BALB} / \mathrm{c}$ splenocytes than $\mathrm{C} 57 \mathrm{Bl} / 6$ splenocytes to live $B$. pseudomallei infection in vitro. The discrepancy in IL-10 production could be explained by the different systems used in the model used by these authors (in vitro) and our model (in vivo), the latter being a closer and more accurate representation of human melioidosis. The lack, or inadequate production, of anti-inflammatory responses in our BALB/C mouse model could also contribute to the development of acute infection, unlike in the $\mathrm{C} 57 \mathrm{Bl} / 6$ mouse model, where the subclinical infection is correlated with a more balanced and modest production of pro- and anti-inflammatory cytokines. It is noteworthy that greater infiltration and accumulation of neutrophils were observed in organs of $\mathrm{BALB} / \mathrm{c}$ mice, whereas early lesions in $\mathrm{C} 57 \mathrm{Bl} / 6$ mice were predominantly comprised of macrophages (Barnes et al., 2001). This observation correlates with the acute inflammation seen in $\mathrm{BALB} / \mathrm{c}$ mice and the better control of infection, possibly by macrophages, in $\mathrm{C} 57 \mathrm{Bl} / 6$ mice (Gan, 2005). However, data from Easton et al., (2007) demonstrated that neutrophils are critical to the generation of the early proinflammatory cytokine environment in the lungs, and suggested an important cytokine-mediated immunomodulatory role for neutrophils in B. pseudomallei infection.

Because the development of the acute form of this disease is not due to a lack, but rather, an excess of inflammation, our nose-only aerosol model, together with the simultaneous detection of a comprehensive list of cytokine and chemokine responses at the protein level, will be an extremely useful platform, critical for future studies to investigate host interactions with bacterial pathogens and will assist in the development of better and/or novel therapeutic options for melioidosis patients.

\section{ACKNOWLEDGEMENTS}

This study was supported by a grant from Defence Science \& Technology Agency, Singapore.

\section{REFERENCES}

Ashdown, L. R. (1979). An improved screening technique for isolation of Pseudomonas pseudomallei from clinical specimens. Pathology 11, 293-297.

Barnes, J. L. \& Ketheesan, N. (2005). Route of infection in melioidosis. Emerg Infect Dis 11, 638-639.
Barnes, J. L., Ulett, G. C., Ketheesan, N., Clair, T., Summers, P. M. \& Hirst, R. G. (2001). Induction of multiple chemokine and colonystimulating factor genes in experimental Burkholderia pseudomallei infection. Immunol Cell Biol 79, 490-501.

Brown, A. E., Dance, D. A., Suputtamongkol, Y., Chaowagul, W., Kongchareon, S., Webster, H. K. \& White, N. J. (1991). Immune cell activation in melioidosis: increased serum levels of interferon-gamma and soluble interleukin-2 receptors without change in soluble CD8 protein. J Infect Dis 163, 1145-1148.

Car, B. D., Eng, V. M., Schnyder, B., Ozmen, L., Huang, S., Gallay, P., Heumann, D., Aguet, M. \& Ryffel, B. (1994). Interferon gamma receptor deficient mice are resistant to endotoxic shock. J Exp Med 179, 1437-1444.

Cheng, A. C. \& Currie, B. J. (2005). Melioidosis: epidemiology, pathophysiology, and management. Clin Microbiol Rev 18, 383-416.

Cheng, A. C., Jacups, S. P., Gal, D., Mayo, M. \& Currie, B. J. (2006). Extreme weather events and environmental contamination are associated with case-clusters of melioidosis in the Northern Territory of Australia. Int J Epidemiol 35, 323-329.

Currie, B. J. (2003). Melioidosis: an important cause of pneumonia in residents of and travellers returned from endemic regions. Eur Respir J 22, 542-550.

Currie, B. J. \& Jacups, S. P. (2003). Intensity of rainfall and severity of melioidosis, Australia. Emerg Infect Dis 9, 1538-1542.

Easton, A., Haque, A., Chu, K., Lukaszewski, R. \& Bancroft, G. J. (2007). A critical role for neutrophils in resistance to experimental infection with Burkholderia pseudomallei. J Infect Dis 195, 99-107.

Friedland, J. S., Suputtamongkol, Y., Remick, D. G., Chaowagul, W., Strieter, R. M., Kunkel, S. L., White, N. J. \& Griffin, G. E. (1992). Prolonged elevation of interleukin- 8 and interleukin- 6 concentrations in plasma and of leukocyte interleukin- 8 mRNA levels during septicemic and localized Pseudomonas pseudomallei infection. Infect Immun 60, 2402-2408.

Gan, Y. H. (2005). Interaction between Burkholderia pseudomallei and the host immune response: sleeping with the enemy? I Infect Dis 192, 1845-1850.

Guyton, A. C. (1947). Measurement of the respiratory volumes of laboratory animals. Am J Phys 150, 70-77.

Heremans, H., Van Damme, J., Dillen, C., Dijkmans, R. \& Billiau, A. (1990). Interferon gamma, a mediator of lethal lipopolysaccharideinduced Shwartzman-like shock reactions in mice. J Exp Med 171, 18531869.

Hoppe, I., Brenneke, B., Rohde, M., Kreft, A., Haussler, S., Reganzerowski, A. \& Steinmetz, I. (1999). Characterization of a murine model of melioidosis: comparison of different strains of mice. Infect Immun 67, 2891-2900.

Howe, C., Sampath, A. \& Spotnitz, M. (1971). The pseudomallei group: a review. J Infect Dis 124, 598-606.

Jeddeloh, J. A., Fritz, D. L., Waag, D. M., Hartings, J. M. \& Andrews, G. P. (2003). Biodefense-driven murine model of pneumonic melioidosis. Infect Immun 71, 584-587.

Koo, G. C. \& Gan, Y. H. (2006). The innate interferon gamma response of $\mathrm{BALB} / \mathrm{c}$ and $\mathrm{C} 57 \mathrm{BL} / 6$ mice to in vitro Burkholderia pseudomallei infection. BMC Immunol 7, 19.

Lauw, F. N., Simpson, A. J., Prins, J. M., Smith, M. D., Kurimoto, M., van Deventer, S. J., Speelman, P., Chaowagul, W., White, N. J. \& van der Poll, T. (1999). Elevated plasma concentrations of interferon (IFN)gamma and the IFN-gamma-inducing cytokines interleukin (IL)-18, IL12, and IL-15 in severe melioidosis. J Infect Dis 180, 1878-1885.

Leakey, A. K., Ulett, G. C. \& Hirst, R. G. (1998). BALB/c and C57Bl/6 mice infected with virulent Burkholderia pseudomallei provide 
contrasting animal models for the acute and chronic forms of human melioidosis. Microb Pathog 24, 269-275.

Lertmemongkolchai, G., Cai, G., Hunter, C. A. \& Bancroft, G. J. (2001). Bystander activation of CD8 $+\mathrm{T}$ cells contributes to the rapid production of IFN-gamma in response to bacterial pathogens. $J$ Immunol 166, 1097-1105.

Liu, B., Koo, G. C., Yap, E. H., Chua, K. L. \& Gan, Y. H. (2002). Model of differential susceptibility to mucosal Burkholderia pseudomallei infection. Infect Immun 70, 504-511.

Liu, Y., Loh, J. P., Aw, L. T., Yap, E. P., Lee, M. A. \& Ooi, E. E. (2006). Rapid molecular typing of Burkholderia pseudomallei, isolated in an outbreak of melioidosis in Singapore in 2004, based on variablenumber tandem repeats. Trans R Soc Trop Med Hyg 100, 687-692.
Reed, L. J. \& Muench, H. A. (1938). Simple method of estimating fifty per cent endpoints. Am J Hyg 27, 493-497.

Santanirand, P., Harley, V. S., Dance, D. A., Drasar, B. S. \& Bancroft, G. J. (1999). Obligatory role of gamma interferon for host survival in a murine model of infection with Burkholderia pseudomallei. Infect Immun 67, 3593-3600.

Ulett, G. C., Ketheesan, N. \& Hirst, R. G. (2000). Proinflammatory cytokine mRNA responses in experimental Burkholderia pseudomallei infection in mice. Acta Trop 74, 229-234.

Wiersinga, W. J., van der Poll, T., White, N. J., Day, N. P. \& Peacock, S. J. (2006). Melioidosis: insights into the pathogenicity of Burkholderia pseudomallei. Nat Rev Microbiol 4, 272-282. 